\title{
Estimates on translations and Taylor expansions in fractional Sobolev spaces
}

\author{
Félix del Teso* David Gómez-Castro ${ }^{\star \dagger} \quad$ Juan Luis Vázquez ${ }^{\ddagger}$
}

April 28, 2020

\begin{abstract}
In this paper we study how the (normalised) Gagliardo semi-norms $[u]_{W^{s, p}\left(\mathbb{R}^{n}\right)}$ control translations. In particular, we prove that $\|u(\cdot+y)-u\|_{L^{p}\left(\mathbb{R}^{n}\right)} \leq C[u]_{W^{s, p}\left(\mathbb{R}^{n}\right)}|y|^{s}$ for $n \geq 1, s \in[0,1]$ and $p \in[1,+\infty]$, where $C$ depends only on $n$. We then obtain a corresponding higher-order version of this result: we get fractional rates of the error term in the Taylor expansion. We also present relevant implications of our two results. First, we obtain a direct proof of several compact embedding of $W^{s, p}\left(\mathbb{R}^{n}\right)$ where the Fréchet-Kolmogorov Theorem is applied with known rates. We also derive fractional rates of convergence of the convolution of a function with suitable mollifiers. Thirdly, we obtain fractional rates of convergence of finite-difference discretizations for $W^{s, p}\left(\mathbb{R}^{n}\right)$.
\end{abstract}

2020 Mathematics Subject Classification. 46E35, 26D10.

Keywords: Fractional Sobolev spaces, Gagliardo norms, translation estimates, Taylor expansions, discretization error, interpolation, convolution.

\section{Introduction and main results}

It is a classical result that for every $u \in W^{1,1}\left(\mathbb{R}^{n}\right)$ the following translation estimate holds:

$$
\|u(\cdot+y)-u\|_{L^{p}\left(\mathbb{R}^{n}\right)} \leq\|\nabla u\|_{L^{p}\left(\mathbb{R}^{n}\right)}|y| .
$$

This result has deep implications which are well-known. First, the compact embedding of $W^{1, p}$ in $L^{p}$ via the Fréchet-Kolmogorov Theorem. A second well-known application is the estimation of the remainder of the Taylor polynomial. This is a key tool used to obtain optimal orders of the convergence of convolutions as well as consistency of finite-difference discretizations. Many other applications can also be found in the literature.

The aim of this paper is to revisit such a theory in the context of fractional Sobolev spaces (which are conveniently presented e.g. in $[1,21,24])$. It is common to define the fractional Sobolev spaces $W^{s, p}\left(\mathbb{R}^{n}\right)$ in the Sobolev-Slobodeckii form. Thus, for $s \in(0,1)$ and $p \in[1, \infty)$ we define the normalised Gagliardo seminorm

$$
[u]_{W^{s, p}\left(\mathbb{R}^{n}\right)}=\left(s(1-s) \int_{\mathbb{R}^{n}} \int_{\mathbb{R}^{n}} \frac{|u(x)-u(y)|^{p}}{|x-y|^{n+s p}} \mathrm{~d} x \mathrm{~d} y\right)^{\frac{1}{p}}
$$

and then $W^{s, p}\left(\mathbb{R}^{n}\right):=\left\{u \in L^{p}\left(\mathbb{R}^{n}\right):[u]_{W^{s, p}\left(\mathbb{R}^{n}\right)}<+\infty\right\}$, which is a Banach space with the norm

$$
\|u\|_{W^{s, p}\left(\mathbb{R}^{n}\right)}=\|u\|_{L^{p}\left(\mathbb{R}^{n}\right)}+[u]_{W^{s, p}\left(\mathbb{R}^{n}\right)} .
$$

The limit cases $s=0, s=1$ for $p \in[1, \infty]$ and $p=\infty$ for $s \in(0,1)$ are defined respectively by

$$
[u]_{W^{0, p}\left(\mathbb{R}^{n}\right)}=\|u\|_{L^{p}\left(\mathbb{R}^{n}\right)},[u]_{W^{1, p}\left(\mathbb{R}^{n}\right)}=\|\nabla u\|_{L^{p}\left(\mathbb{R}^{n}\right)}, \text { and }[u]_{W^{s, \infty}\left(\mathbb{R}^{n}\right)}=\operatorname{ess~sup}_{x, y \in \mathbb{R}^{n}} \frac{|u(x)-u(y)|}{|x-y|^{s}} .
$$

\footnotetext{
^Dpto. de Análisis Matemático y Matemática Aplicada, Universidad Complutense de Madrid. fdelteso@ucm.es

${ }^{\dagger}$ Instituto de Matemática Interdisciplinar, Universidad Complutense de Madrid. dgcastro@ucm.es

${ }^{\ddagger}$ Departamento de Matemáticas, Universidad Autónoma de Madrid. juanluis.vazquez@uam.es
} 
The basic result of this paper asserts that the translation estimate holds in the following fractional Sobolev setting.

Theorem 1.1. The following estimate holds for every $u \in W^{s, p}\left(\mathbb{R}^{n}\right)$ with $p \in[1,+\infty]$ and $s \in[0,1]$ :

$$
\|u(\cdot+y)-u\|_{L^{p}\left(\mathbb{R}^{n}\right)} \leq C[u]_{W^{s, p}\left(\mathbb{R}^{n}\right)}|y|^{s} .
$$

The constant $C>0$ depends only $n$, and not on $s$ or $p$. An admissible value is $C=4 n(n+1) e^{\frac{1}{e \omega_{n} n}}$.

Let us briefly comment on previous results related to Theorem 1.1. This kind of translation estimate for $n=1$ can be found in Simon's [23, Lemma 5] in an abstract setting (vector valued functions) and without explicit bounds on the constants. Like in [23], the technique of our proof is based on $K$-interpolation theory (which we introduce in detail in Section 3). On the other hand, the result

$$
\|u(\cdot+y)-u\|_{L^{p}\left(\mathbb{R}^{n}\right)} \leq s^{-\frac{1}{p}} C(n, p)[u]_{W^{s, p}\left(\mathbb{R}^{n}\right)}|y|^{s}
$$

was obtained for $p=1$ by Ambrosio-de Philippis-Martinazzi [3, Proposition 4] and for $p \in(1, \infty)$ by Brasco-Lindgren-Parini [6, Lemma A.1] via direct computations. As we recall in Section 1.1, the normalised Gagliardo seminorm converges to the integer seminorm up to a constant. Hence, [3, 6] do not provide uniform estimates as $s \rightarrow 0$. However, it seems very natural to include this limit case since for $s=0$ we have the simple rule $\|u(\cdot+y)-u\|_{L^{p}\left(\mathbb{R}^{n}\right)} \leq 2\|u\|_{L^{p}\left(\mathbb{R}^{n}\right)}$. We recall that these results can also be understood as embeddings of Besov spaces (see a detailed comment in Section 1.2).

Compared to previous related literature, a novel contribution of Theorem 1.1 is the fact that we obtain a clean statement with a constant $C$ which is uniform on the whole ranges $s \in[0,1]$ and $p \in[1, \infty]$ (see Section 1.1). Essentially, this is done by recalling a sharp equivalence between the Gagliardo seminorm and the norm in some interpolation spaces given in the recent paper by Brasco and Salort [7]. The control of the constants in inequalities like ours can be important in the applications.

Once Theorem 1.1 is established, we can prove the corresponding result with higher-order Sobolev regularity. It is well known that a function $u$ in the Hölder space $C^{k, s}\left(\mathbb{R}^{n}\right)$ for some $k \geq 0$ integer and some $s \in[0,1]$ satisfies

$$
\left\|u(\cdot+y)-P_{k} u(\cdot, y)\right\|_{L^{\infty}\left(\mathbb{R}^{n}\right)} \leq C[u]_{C^{k, s}\left(\mathbb{R}^{n}\right)}|y|^{k+s} .
$$

where $C=C(k)$ is a positive constant and $P_{k} u$ denotes the Taylor polynomial of order $k$ and centred at $x$ of the function $u$, i.e

$$
P_{k} u(x, y):=\sum_{|\alpha| \leq k} \frac{D^{\alpha} u(x)}{\alpha !} y^{\alpha},
$$

where we are using the standard multi-index notation (see (1.3) below). The higher-order fractional Sobolev norms for $k \in \mathbb{N}$ and $s \in(0,1]$ are defined as

$$
\|u\|_{W^{k+s, p}\left(\mathbb{R}^{n}\right)}:=\|u\|_{W^{k, p}\left(\mathbb{R}^{n}\right)}+[u]_{W^{k+s, p}\left(\mathbb{R}^{n}\right)}, \quad \text { where } \quad[u]_{W^{k+s, p}\left(\mathbb{R}^{n}\right)}:=\max _{|\alpha|=k}\left[D^{\alpha} u\right]_{W^{s, p}\left(\mathbb{R}^{n}\right)}
$$

and $W^{k+s, p}\left(\mathbb{R}^{n}\right):=\left\{u \in W^{k, p}\left(\mathbb{R}^{n}\right):[u]_{W^{k+s, p}\left(\mathbb{R}^{n}\right)}<+\infty\right\}$. For $p=\infty$ they coincide with the Hölder spaces: for $k \geq 0$ integer and $s \in(0,1)$ we have $W^{k+s, \infty}\left(\mathbb{R}^{n}\right)=C^{k, s}\left(\mathbb{R}^{n}\right)$.

In this higher-order fractional Sobolev setting, we prove the following estimate of the remainder term in the Taylor expansion.

Theorem 1.2. Let $u \in W^{k+s, p}\left(\mathbb{R}^{n}\right)$ where $p \in[1, \infty], s \in[0,1]$, and $k \geq 0$ is an integer. The following estimate holds

$$
\left\|u(\cdot+y)-P_{k}(\cdot, y)\right\|_{L^{p}\left(\mathbb{R}^{n}\right)} \leq C[u]_{W^{k+s, p}\left(\mathbb{R}^{n}\right)}|y|^{k+s},
$$

where $C>0$ depends only on $n$ and $k$.

Notation. Throughout the paper, $\omega_{n}$ denotes the volume of the unit ball of $\mathbb{R}^{n}$ and we will use the multi-index notation $\alpha=\left(\alpha_{1}, \ldots, \alpha_{n}\right) \in \mathbb{N}^{n}, x=\left(y_{1}, \ldots, y_{n}\right) \in \mathbb{R}^{n}$,

$$
|\alpha|=\sum_{i=1}^{n} \alpha_{i}, \quad \alpha !=\prod_{i=1}^{n} \alpha_{i} !, \quad y^{\alpha}=\prod_{i=1}^{n} y_{i}^{\alpha_{i}}, \quad \text { and } \quad D^{\alpha} u=\frac{\partial^{|\alpha|} u}{\partial x_{1}^{\alpha_{1}} \cdots x_{n}^{\alpha_{n}}} .
$$


Structure of the paper. In the rest of Section 1 we make some comments on relevant results that in a way or another intersect and complement ours. In Section 2 we present several applications of Theorem 1.2. We delay the proof of the main theorems to Section 3. We conclude the paper with a list of comments and extensions in Section 4.

\subsection{Comments on the limits as $s \rightarrow 0^{+}$and $s \rightarrow 1^{-}$}

Let us note that for $p \in[1,+\infty)$ and $u \in C_{c}^{\infty}\left(\mathbb{R}^{n}\right)$ the normalised Gagliardo seminorm converges to classical seminorms up to constants

$$
\begin{array}{ll}
{[u]_{W^{s, p}\left(\mathbb{R}^{n}\right)} \rightarrow K_{1}\|\nabla u\|_{L^{p}\left(\mathbb{R}^{n}\right)}} & \text { as } s \rightarrow 1^{-} \\
{[u]_{W^{s, p}\left(\mathbb{R}^{n}\right)} \rightarrow K_{0}\|u\|_{L^{p}\left(\mathbb{R}^{n}\right)}} & \text { as } s \rightarrow 0^{+}
\end{array}
$$

where the different constants $K_{i}=K_{i}(n, p)$ are positive (see Bourgain-Brezis-Mironescu in [5, Corollary 2] and Maz'ya-Shaposhnikova in [20, Theorem 3]). Hence, as $s$ approaches 1, we recover (1.1) up to a constant. Dávila [14] showed a similar result for $u \in B V(\Omega)$. This kind of interpolation norm convergence at the endpoints of the interpolation is discussed in a general setting in [22].

\subsection{Related known results in Besov spaces}

The Besov spaces $B_{p, \infty}^{s}\left(\mathbb{R}^{n}\right)$ can be defined as the functions in $L^{p}\left(\mathbb{R}^{n}\right)$ such that $\|u(\cdot+y)-u\|_{L^{p}} \leq C|y|^{s}$ for some constant $C$. Hence, Theorem 1.1 implies, in particular, that $W^{s, p}\left(\mathbb{R}^{n}\right) \subset B_{p, \infty}^{s}\left(\mathbb{R}^{n}\right)$.

There are two equivalent ways of defining the Besov spaces $B_{p, q}^{s}\left(\mathbb{R}^{n}\right)$. The first one is in terms of the integrability of the $p$-modulus of continuity as presented in [4]. More precisely, for $s \in(0,1]$, we take $\omega_{p}(u, t)=\sup _{|y| \leq t}\|u(\cdot+y)-u\|_{L^{p}\left(\mathbb{R}^{n}\right)}$ and define the Besov space $B_{p, q}^{s}\left(\mathbb{R}^{n}\right)$ as the functions in $L^{p}\left(\mathbb{R}^{n}\right)$ with finite Besov norm

$$
\|u\|_{B_{p, q}^{s}\left(\mathbb{R}^{n}\right)}= \begin{cases}\|u\|_{L^{p}\left(\mathbb{R}^{n}\right)}+\left(\int_{0}^{\infty}\left(t^{-s} \omega_{p}(u, t)\right)^{q} \frac{d t}{t}\right)^{\frac{1}{q}} & \text { for } \quad q<\infty \\ \|u\|_{L^{p}\left(\mathbb{R}^{n}\right)}+\sup _{t>0}\left(t^{-s} \omega_{p}(u, t)\right) & \text { for } \quad q=\infty\end{cases}
$$

This norm is fairly similar to the Gagliardo norm in fractional Sobolev spaces, and in fact it is known that $W^{s, p}\left(\mathbb{R}^{n}\right)=B_{p, p}^{s}\left(\mathbb{R}^{n}\right)$. Note also that a version of (1.2) can be written in terms of $p$-modulus as

$$
\sup _{t>0}\left(t^{-s} \omega_{p}(u, t)\right) \leq C[u]_{W^{s, p}\left(\mathbb{R}^{n}\right)}
$$

This definition of Besov spaces when $s>1$ requires the higher order $p$-modulus of continuity, for which we refer the reader to [4, Section 4 in Chapter 5].

The second definition (see [1,24]), valid for $q<+\infty$, is given in terms of $K$-interpolation (that will be properly introduced in Section 3) as

$$
B_{p, q}^{s}\left(\mathbb{R}^{n}\right)=\left(L^{p}\left(\mathbb{R}^{n}\right), W^{k, p}\left(\mathbb{R}^{n}\right)\right)_{\frac{s}{k}, q ; K}
$$

where $k$ is a positive integer greater than $s$. The equivalence of both definitions is proven in any of the references (see, e.g., [1, Theorem 7.47] or [24, Chapter 35]).

In fact, for the interpolation is known (see [1, Corollary 7.17]) that $\left(X_{0}, X_{1}\right)_{\theta, p ; K} \subset\left(X_{0}, X_{1}\right)_{\theta, q ; K}$ if $1 \leq p \leq q \leq \infty$ and $\theta \in(0,1)$. Hence, $W^{s, p}\left(\mathbb{R}^{n}\right) \subset B_{p, \infty}^{s}\left(\mathbb{R}^{n}\right)$ can also be deduced in this way. Embeddings between Besov spaces are discussed at length in [4]. Actually, through the mentioned embeddings, we can retrieve (1.2) but this time with an unknown constant $C(n, p, s)$ and depending on the $W^{s, p}$ norm (not the Gagliardo seminorm). To the best of our knowledge, it seems that (1.2) is not widely-known. In practice, many authors use weaker, more difficult and less powerful properties of the fractional Sobolev spaces. We provide a clear and direct statement and proof of (1.2) with explicit constants. 


\section{Applications}

We devote this section to present several direct applications of the above results which motivated our investigation.

\subsection{Rellich-Kondrachov. Compact embeddings of $W^{s, p}\left(\mathbb{R}^{n}\right)$}

First, we prove the compact embedding of $W^{s, p}\left(\mathbb{R}^{n}\right)$ more directly and with further generality than in previous literature (cf. [17, Section 7]).

Theorem 2.1 (Fractional Rellich-Kondrachov). Let $p \in[1, \infty)$ and $\Omega \subset \mathbb{R}^{n}$ be measurable with finite measure and $K \subset \mathbb{R}^{n}$ compact. For $s \in(0,1)$ We have the following:

1. If $s p<n$ then $W^{s, p}\left(\mathbb{R}^{n}\right)$ is compactly embedded in $L^{q}(\Omega)$ for $q<p^{\star}(n, s):=\frac{n p}{n-s p}$.

2. If $s p=n$ then $W^{s, p}\left(\mathbb{R}^{n}\right)$ is compactly embedded in $L^{q}(\Omega)$ for $q<\infty$.

3. If $s p>n$ then $W^{s, p}\left(\mathbb{R}^{n}\right)$ is compactly embedded in $C^{\beta}(K)$ for any with $\beta<s-\frac{n}{p}$.

Furthermore, if $\Omega$ is a bounded with Lipschitz boundary, then

4. If $s>t$ then $W^{s, p}\left(\mathbb{R}^{n}\right)$ is compactly embedded in $W^{t, p}(\Omega)$.

More general results in which $W^{t, p}$ is replaced by $W^{t, q}$ for different values of $q$ can be obtained in a similar fashion. We recall the fractional Gagliardo-Nirenberg interpolation inequality that can be found in [9]: if $\Omega$ is a standard domain (i.e. $\mathbb{R}^{n}$, a half-space or it is bounded with Lipschitz boundary) then

$$
\|f\|_{W^{s, p}(\Omega)} \leq C\|f\|_{W^{s_{1}, p_{1}(\Omega)}}^{\theta}\|f\|_{W^{s_{2}, p_{2}(\Omega)}}^{1-\theta}, \quad \theta \in(0,1), \quad s=\theta s_{1}+(1-\theta) s_{2}, \quad \frac{1}{p}=\frac{\theta}{p_{1}}+\frac{1-\theta}{p_{2}} .
$$

as long at it fails that: $s_{2}$ is an integer $\geq 1$ and $p_{2}=1$ and $s_{2}-s_{1} \leq 1-\frac{1}{p_{1}}$.

The fractional version of the Gagliardo-Nirenberg-Sobolev inequality for $s \in(0,1)$ can be found in [17], where the compact embedding in Item 1 in Theorem 2.1 and the continuous are proved for the rest of the cases. In [17, Theorem 6.5] for $s p<n$

$$
\|u\|_{L^{p^{\star}\left(\mathbb{R}^{n}\right)}} \leq C[u]_{W^{s, p}\left(\mathbb{R}^{n}\right)}, \quad \text { where } p^{\star}=p^{\star}(n, s),
$$

for $s p=n$ in $[17$, Theorem 6.9$]$

$$
\|u\|_{L^{q\left(\mathbb{R}^{n}\right)}} \leq C\|u\|_{W^{s, p}\left(\mathbb{R}^{n}\right)}, \quad \text { for all } q \in[p,+\infty),
$$

and if $s p>n$ we have in $[17$, Theorem 8.2] that

$$
\|u\|_{C^{0, \alpha}\left(\mathbb{R}^{n}\right)} \leq C\|u\|_{W^{s, p}\left(\mathbb{R}^{n}\right)}, \quad \text { for all } \alpha \leq s-\frac{n}{p} .
$$

For $s \in(0,1]$ and $s p>n$ it also worth mentioning that the inequality above holds in terms of the semi-norms in what it typically known as Morrey's inequality

$$
|u(x)-u(y)| \leq C[u]_{W^{s, p}\left(\mathbb{R}^{n}\right)}|x-y|^{s-\frac{n}{p}} .
$$

The proof for $s=1$ is classical and for $s \in(0,1)$, it may be found in [19, Proposition 14.40 and Corollary 14.28] using Besov spaces (see also [6] for a more direct proof). For the sake of completeness, for $s \in(0,1)$ and $p \in[1, \infty)$ we recall the fractional Poincaré inequality (see [18, Proposition 2.1])

$$
\left\|u-\frac{1}{|\Omega|} \int_{\Omega} u(x) \mathrm{d} x\right\|_{L^{p}(\Omega)} \leq \frac{\operatorname{diam}(\Omega)^{s+\frac{n}{p}}}{|\Omega|^{\frac{1}{p}}} \frac{[u]_{W^{s, p}\left(\mathbb{R}^{n}\right)}}{s^{\frac{1}{p}}(1-s)^{\frac{1}{p}}}
$$

We recall a particular form of the Fréchet-Kolmogorov Theorem (see, e.g., Theorem 4.26 in [8])

Theorem 2.2. Let $\mathcal{F}$ be a bounded set in $L^{p}\left(\mathbb{R}^{n}\right)$ for $p \in[1, \infty)$ and assume

$$
\text { for every } \varepsilon>0 \text {, there exists } \delta>0 \text { such that }\|f(\cdot+y)-f\|_{L^{p}\left(\mathbb{R}^{n}\right)} \leq \varepsilon, \forall|y|<\delta \text { and } f \in \mathcal{F} \text {. }
$$

Then, for every $\Omega \subset \mathbb{R}^{n}$ measurable of finite measure the closure of $\left.\mathcal{F}\right|_{\Omega}$ is compact in $L^{p}(\Omega)$. 
With this result, we can proceed to the proof of Theorem 2.1. Once (1.2) is established, we rely on standard arguments. For similar proofs of Item 1 see [17, Theorem 6.5] or [6, Theorem 2.7] which follow the usual argument, see e.g. [8, Theorem 9.16]).

Proof of Theorem 2.1. First, let us prove the the result for $q=p$. We take as $\mathcal{F}$ any bounded set of $W^{s, p}\left(\mathbb{R}^{n}\right)$. Naturally, it is bounded in $L^{p}\left(\mathbb{R}^{n}\right)$. By Theorem 1.1 we have that

$$
\|f(\cdot+y)-f\|_{L^{p}\left(\mathbb{R}^{n}\right)} \leq C\|f\|_{W^{s, p}\left(\mathbb{R}^{n}\right)}|y|^{s} \leq \tilde{C}|y|^{s} \quad \text { with } \quad \tilde{C}=C \sup _{f \in \mathcal{F}}\left\{\|f\|_{W^{s, p}\left(\mathbb{R}^{n}\right)}\right\}
$$

for any $f \in \mathcal{F}$. Given $\varepsilon>0$, we can choose $\delta=(\varepsilon / \tilde{C})^{\frac{1}{s}}$ so that $(2.4)$ holds. Applying Theorem 2.2 we conclude that $W^{s, p}\left(\mathbb{R}^{n}\right)$ is compactly embedded in $L^{p}(\Omega)$.

For the rest of the cases we prove sequential compactness. Let $u_{m} \in W^{s, p}\left(\mathbb{R}^{n}\right)$ be a bounded sequence.

1) For $q \leq p$ the result is trivial by the first part. Let $q \in\left[p, p^{\star}\right)$. We prove sequential compactness. Let $u_{m}$ be a bounded sequence in $W^{s, p}\left(\mathbb{R}^{n}\right)$. By the previous part is has a subsequence, still denoted $u_{m}$, that converges strongly in $L^{p}(\Omega)$. By Hölder's inequality

$$
\left\|u_{m}-u_{k}\right\|_{L^{q}(\Omega)} \leq\left\|u_{m}-u_{k}\right\|_{L^{p}(\Omega)}^{1-\theta}\left\|u_{m}-u_{k}\right\|_{L^{p^{\star}}(\Omega)}^{\theta} \leq 2\left\|u_{m}-u_{k}\right\|_{L^{p}(\Omega)}^{1-\theta} \sup _{\ell}\left\|u_{\ell}\right\|_{L^{p^{\star}}(\Omega)}^{\theta}
$$

Hence, due to the continuous embedding (2.2) and the fact that $u_{m}$ is a Cauchy sequence in $L^{p}(\Omega)$, we have that $u_{m}$ is a Cauchy sequence in $L^{q}(\Omega)$. Hence, it converges strongly in $L^{q}(\Omega)$.

2) The same argument applies.

3) We apply (2.3) and the Ascoli-Arzelá theorem.

4) In this case, there exists a subsequence, still denote $u_{m}$ such that $u_{m} \rightarrow u$ in $L^{p}\left(\mathbb{R}^{n}\right)$. We can apply (2.1) with $s_{1}=s, s_{2}=0, \theta=t / s, p_{0}=p_{1}=p$ and write

$$
\left\|u_{m}-u_{n}\right\|_{W^{t, p}(\Omega)} \leq C\left\|u_{m}-u_{n}\right\|_{W^{s, p}\left(\mathbb{R}^{n}\right)}^{\theta}\left\|u_{m}-u_{n}\right\|_{L^{p}(\Omega)}^{1-\theta} \leq C\left\|u_{m}-u_{n}\right\|_{L^{p}(\Omega)}^{1-\theta} .
$$

Hence $u_{m}$ is Cauchy in $W^{t, p}(\Omega)$.

Remark 1. A natural question is whether these embeddings are also compact into $L^{p}\left(\mathbb{R}^{n}\right)$. As in the integer case, this is not true. A very simple counterexample is the following. Let $u \in C_{c}^{\infty}\left(\mathbb{R}^{n}\right) \backslash\{0\}$ and consider the sequence $u_{k}(x)=u\left(x-k e_{1}\right)$ where $k \in \mathbb{N}$. Clearly $\left\|u_{k}\right\|_{W^{s, p}\left(\mathbb{R}^{n}\right)}=\|u\|_{W^{s, p}\left(\mathbb{R}^{n}\right)}$ so it is bounded. The weak limit of $u_{k}$ in $L^{p}\left(\mathbb{R}^{n}\right)$ is clearly 0 . Hence, the sequence cannot have an $L^{p}$-strongly convergence subsequence.

The Fréchet-Kolmogorov Theorem states that a family $\mathcal{F}$ is relatively compact in $L^{p}\left(\mathbb{R}^{n}\right)$ if and only if it is bounded in $L^{p}\left(\mathbb{R}^{n}\right)$ and the following hold:

1. The tails are uniformly controlled, i.e. for every $\varepsilon>0$ there exists $R$ such that

$$
\int_{|x|>R}|f|^{p} \leq \varepsilon, \quad \forall f \in \mathcal{F}
$$

2. The translations are uniformly controlled, i.e. (2.4).

The boundedness of the tails cannot be uniformly controlled by the $W^{s, p}$ norm (as in the counterexample), and hence the weaker form Theorem 2.2 is more convenient.

The reader may find in [12] and [13] an interesting proof of the continuous embeddings between fractional Sobolev spaces.

\subsection{Rates of convergence of convolutions for $W^{s, p}\left(\mathbb{R}^{n}\right)$ functions}

A well-know result says that if $u \in L^{p}\left(\mathbb{R}^{n}\right)$ and the $\rho_{\varepsilon}$ form a suitable family of mollifiers, then $\rho_{\varepsilon} * u \rightarrow u$ in $L^{p}\left(\mathbb{R}^{n}\right)$. This fact is used in countless theoretical results (for example the proof of Theorem 2.2). However, for certain applications it is relevant to give precise rates of convergence of these convolutions. 
Results for $W^{1, p}\left(\mathbb{R}^{n}\right)$ and $W^{2, p}\left(\mathbb{R}^{n}\right)$ are well-known. The argument is also well known in some fractional Besov spaces.

As a second application of our main result, we recover fractional rates of convergence of convolutions, up to a quadratic order. Notice that the result is better for even mollifiers.

Theorem 2.3. Let $\rho: \mathbb{R}^{n} \rightarrow \mathbb{R}$ is such that $\int_{\mathbb{R}^{n}} \rho(x) \mathrm{d} x=1$ and $\operatorname{supp} \rho=B_{1}$, and let $\rho_{\varepsilon}(x)=\varepsilon^{-n} \rho(x / \varepsilon)$. There exists $C=C(n)$ such that, for every $u \in W^{s, p}\left(\mathbb{R}^{n}\right)$ with $p \in[1, \infty]$ and $s \in[0,1]$, we have

$$
\left\|\rho_{\varepsilon} * u-u\right\|_{L^{p}\left(\mathbb{R}^{n}\right)} \leq C[u]_{W^{s, p}\left(\mathbb{R}^{n}\right)} \varepsilon^{s} .
$$

Furthermore, if $\rho$ is even (i.e. $\rho(y)=\rho(-y)$ ) the result holds for $s \in[0,2]$.

Proof. Consider first the case $s \in[0,1]$. Then, by Jensen's inequality and Theorem 1.1 we have

$$
\begin{aligned}
\left\|\rho_{\varepsilon} * u-u\right\|_{L^{p}\left(\mathbb{R}^{n}\right)}^{p} & =\int_{\mathbb{R}^{n}}\left|\int_{B_{\varepsilon}}(u(x+y)-u(x)) \rho_{\varepsilon}(y) \mathrm{d} y\right|^{p} \mathrm{~d} x \\
& \leq \int_{B_{\varepsilon}} \rho_{\varepsilon}(y) \int_{\mathbb{R}^{n}}|u(x+y)-u(x)|^{p} \mathrm{~d} x \mathrm{~d} y \\
& \leq \sup _{|y| \leq \varepsilon}\|u(\cdot+y)-u\|_{L^{p}\left(\mathbb{R}^{n}\right)}^{p} \leq c^{p} \varepsilon^{s p}[u]_{W^{s, p}\left(\mathbb{R}^{n}\right)}^{p} .
\end{aligned}
$$

Now, let $s \in(1,2]$. Since $\rho$ is even we have that

$$
\int_{\mathbb{R}^{n}} \nabla u(x) \cdot y \rho_{\varepsilon}(y) \mathrm{d} y=\nabla u(x) \cdot \int_{\mathbb{R}^{n}} y \rho_{\varepsilon}(y) \mathrm{d} y=0 .
$$

Hence, we can introduce the extra term $(\nabla u(x) \cdot y) \rho_{\varepsilon}(y)$ in the first inequality of $(2.5)$. Again, Jensen's inequality implies,

$$
\begin{aligned}
\left\|\rho_{\varepsilon} * u-u\right\|_{L^{p}\left(\mathbb{R}^{n}\right)}^{p} & =\int_{\mathbb{R}^{n}}\left|\int_{B_{\varepsilon}}(u(x+y)-u(x)-\nabla u(x) \cdot y) \rho_{\varepsilon}(y) \mathrm{d} y\right|^{p} \mathrm{~d} x \\
& \leq \sup _{|y| \leq \varepsilon}\left\|u(\cdot+y)-P_{1} u(\cdot, y)\right\|_{L^{p}\left(\mathbb{R}^{n}\right)}^{p} .
\end{aligned}
$$

which allows us to conclude the desired result by using Theorem 1.2 with $k=1$ and $t=s-1$. The limit cases are obtained in a similar way.

Remark 2. The conclusion of Theorem 2.3 also holds when $\rho \in L^{1}\left(\mathbb{R}^{n}\right)$ is such that maybe $\|\rho\|_{L^{1}\left(\mathbb{R}^{n}\right)} \neq 1$, $\operatorname{supp} \rho \subset B_{R}$ and it changes signs, and the result reads

$$
\left\|\rho_{\varepsilon} * u-u \int_{\mathbb{R}^{n}} \rho(x) \mathrm{d} x\right\|_{L^{p}\left(\mathbb{R}^{n}\right)} \leq C\|\rho\|_{L^{1}\left(\mathbb{R}^{n}\right)}[u]_{W^{s, p}\left(\mathbb{R}^{n}\right)} \varepsilon^{s},
$$

where $C$ also depends on $R$.

\subsection{Rates of convergence of finite differences for $W^{s, p}\left(\mathbb{R}^{n}\right)$ functions}

One of the most classical numerical methods to solve differential equations is given by finite differences. This method approximates the derivatives by pointwise evaluations of the function itself. The error of this approximation is sometimes referred by the name of consistency of the discretization. Estimates for this error in terms of integer derivatives are easily obtained from the Taylor expansion, and hence such a theory is well-known for $W^{k, p}\left(\mathbb{R}^{n}\right)$ with $k \in \mathbb{N}$.

In the recent literature, there has been significant interest in the study of PDEs of non-local or fractional type, in which the solution usually only lies in a fractional Sobolev class of regularity $W^{s, p}$. This leads to be interested in the study of the consistency of this discrete derivatives in the fractional Sobolev setting. It has been shown (see, e.g. $[11,15])$ that numerical discretizations for this kind of fractional problems can be constructed as fractional powers of this local finite-difference approximations. We obtain the following fractional consistency estimates: 
Theorem 2.4. Let $u \in W^{s, p}\left(\mathbb{R}^{n}\right)$ and $p \in(1, \infty)$. We have that

(a) If $s \in(1,2]$ then

$$
\left\|\frac{u\left(\cdot+e_{i} h\right)-u}{h}-\frac{\partial u}{\partial x_{i}}\right\|_{L^{p}\left(\mathbb{R}^{n}\right)} \leq c[u]_{W^{s, p}\left(\mathbb{R}^{n}\right)} h^{s-1} .
$$

(b) If $s \in(2,4]$ then

$$
\left\|\frac{u\left(\cdot+e_{i} h\right)+u\left(\cdot-e_{i} h\right)-2 u}{h^{2}}-\frac{\partial^{2} u}{\partial x_{i}^{2}}\right\|_{L^{p}\left(\mathbb{R}^{n}\right)} \leq c[u]_{W^{s, p}\left(\mathbb{R}^{n}\right)} h^{s-2} .
$$

Here, $\left\{e_{i}\right\}_{i=1}^{n}$ denotes the standard basis of $\mathbb{R}^{n}$.

Proof of Theorem 2.4. We prove the result for $u \in C_{c}^{\infty}\left(\mathbb{R}^{n}\right)$, and the result holds in general by approximation. First, we point out that (a) is precisely Theorem 1.2 with $k=1, t=s-1$ and $y=h e_{i}$ and then dividing by $h$. For (b) we note that

$$
u\left(x \pm e_{i} h\right)=u(x) \pm h \frac{\partial u}{\partial x_{i}}(x)+\frac{h^{2}}{2} \frac{\partial^{2} u}{\partial x_{i}^{2}}(x) \pm \frac{h^{3}}{6} \frac{\partial^{3} u}{\partial x_{i}^{3}}(x)+R_{ \pm}(x)
$$

where, by Theorem 1.2, we have $\left\|R_{ \pm}(x)\right\|_{L^{p}\left(\mathbb{R}^{n}\right)} \leq C h^{s}[u]_{W^{s, p}\left(\mathbb{R}^{n}\right)}$ for $s \in(2,4]$. Hence

$$
\frac{u\left(x+e_{i} h\right)+u\left(x-e_{i} h\right)-2 u(x)}{h^{2}}-\frac{\partial^{2} u}{\partial x_{i}^{2}}(x)=\frac{1}{h^{2}}\left(R_{+}(x)-R_{-}(x)\right) .
$$

This completes the proof.

Remark 3. Applying these techniques, one can recover similar estimates for any of the classical finitedifference approximations.

\section{Proof of the main results}

The main technique we will use in the proof of Theorem 1.1 is the theory of interpolation spaces. Interpolation techniques are delicate and are sometimes used carelessly. In order to be very precise, we will introduce several auxiliary definitions. For $s>0$, we can define $W^{s, p}\left(\mathbb{R}^{n}\right)$ as the closure of $C_{c}^{\infty}\left(\mathbb{R}^{n}\right)$ in $L^{p}\left(\mathbb{R}^{n}\right)$ with respect to the $\|\cdot\|_{W^{s, p}\left(\mathbb{R}^{n}\right)}$ norm, or equivalently

$$
W^{s, p}\left(\mathbb{R}^{n}\right)=\left\{u \in L^{p}\left(\mathbb{R}^{n}\right):\|u\|_{W^{s, p}\left(\mathbb{R}^{n}\right)}<+\infty\right\} .
$$

The $K$-interpolation space for $s \in(0,1)$ and $p \in[1, \infty)$ of two spaces $X_{0}$ and $X_{1}$ is defined as the elements in $X_{0}+X_{1}$ which have finite (normalised) $K$-interpolation norm

$$
\|u\|_{\left(X_{0}, X_{1}\right)_{s, p ; K}}:=\left(s(1-s) \int_{0}^{+\infty}\left(\frac{K(t, u)}{t^{s}}\right)^{p} \frac{\mathrm{d} t}{t}\right)^{\frac{1}{p}},
$$

where

$$
K(t, u)=K\left(t, u, X_{0}, X_{1}\right):=\inf \left\{\left\|u_{0}\right\|_{X_{0}}+t\left\|u_{1}\right\|_{X_{1}}: u=u_{0}+u_{1}, u_{0} \in X_{0}, u_{1} \in X_{1}\right\} .
$$

In order for this definition to make sense, $X_{0} \cap X_{1}$ must be nontrivial and $X_{0}+X_{1}$ must be a topological vector space. It is then said that $\left\{X_{0}, X_{1}\right\}$ is an interpolation pair. Notice that, for each $s$, removing $s(1-s)$ in $(3.1)$ gives an equivalent norm. In fact, most books (e.g. [1, 4]) do not include this constant. However, Milman points out in [22] that the behaviour mentioned in Section 1.1 occurs in general, and this constant $s(1-s)$ allows to pass to the limits as $s \rightarrow 0,1$.

A well known interpolation result asserts that

$$
W^{s, p}\left(\mathbb{R}^{n}\right)=\left(L^{p}\left(\mathbb{R}^{n}\right), W^{1, p}\left(\mathbb{R}^{n}\right)\right)_{s, p ; K}
$$


via the $K$-interpolation (see Section 1.2). A very complete but rather technical presentation of this kind of results can be found in [2]. Let us define for $u \in C_{c}^{\infty}\left(\mathbb{R}^{n}\right)$ the (normalised) interpolation-like semi-norm

$$
\|u\|_{s, p}:=\left(s(1-s) \int_{0}^{+\infty}\left(\frac{\bar{K}(t, u)}{t^{s}}\right)^{p} \frac{\mathrm{d} t}{t}\right)^{\frac{1}{p}}
$$

where

$$
\bar{K}(t, u)=\inf _{u_{1} \in C_{c}^{\infty}\left(\mathbb{R}^{n}\right)}\left\{\left\|u-u_{1}\right\|_{L^{p}\left(\mathbb{R}^{n}\right)}+t\left\|\nabla u_{1}\right\|_{L^{p}\left(\mathbb{R}^{n}\right)}\right\}
$$

In [7] the authors prove that, for $u \in C_{c}^{\infty}\left(\mathbb{R}^{n}\right)$, this norm is equivalent to the (normalised) Gagliardo seminorm

$$
\frac{1}{C}[u]_{W^{s, p}\left(\mathbb{R}^{n}\right)} \leq\|u\|_{s, p} \leq C[u]_{W^{s, p}\left(\mathbb{R}^{n}\right)},
$$

where $C$ depends only on $n$ and $p$.

Remark 4. Some authors define a space $\dot{W}^{s, p}\left(\mathbb{R}^{n}\right)$ as the completion of $C_{c}^{\infty}\left(\mathbb{R}^{n}\right)$ with respect the semi-norm $[\cdot]_{W^{s, p}\left(\mathbb{R}^{n}\right)}$. In these terms, the results in [7] would imply

$$
\dot{W}^{s, p}\left(\mathbb{R}^{n}\right) \simeq\left(L^{p}\left(\mathbb{R}^{n}\right), \dot{W}^{1, p}\left(\mathbb{R}^{n}\right)\right)_{s, p ; K}
$$

However, this introduces some functional analysis difficulties that we want to avoid here. We point out that the completion is a delicate process. Let $s \in(0,1]$ and $s p>n$. Let $\varphi \in C_{c}^{\infty}\left(\mathbb{R}^{n}\right)$ with value 1 in $B_{1}$ and 0 outside $B_{2}$ and $\varphi_{m}(x)=\varphi(x / m)$. By a simple change in variable, it is easy to see that $\left[\varphi_{m}\right]_{W^{s, p}\left(\mathbb{R}^{n}\right)}=m^{\frac{n}{p}-s}[\varphi]_{W^{s, p}\left(\mathbb{R}^{n}\right)}$. We thus get $\varphi_{m} \rightarrow 1$ uniformly over compacts and $\left[\varphi_{m}\right]_{W^{s, p}\left(\mathbb{R}^{n}\right)} \rightarrow 0$. Therefore, in the completion the zero function is equivalent to all constants.

A key step in the proof is to use (3.4) together with the fact that $X_{0}=\left(X_{0}, X_{0}\right)_{s, p ; K}$ and the explicit value of (normalised) interpolation norm is given by

$$
\|u\|_{X_{0}}=p^{\frac{1}{p}}\|u\|_{\left(X_{0}, X_{0}\right)_{s, p ; K}}
$$

(see [22, Section 2.1]) We now proceed to the proof of the main result.

Proof of Theorem 1.1. Case 1: $p=\infty$ and $s \in[0,1]$. This is a classical due to the definition of $W^{s, \infty}\left(\mathbb{R}^{n}\right)$.

From here and in the following cases, we will assume that $u \in C_{c}^{\infty}\left(\mathbb{R}^{n}\right)$ and the results in $W^{s, p}\left(\mathbb{R}^{n}\right)$ hold by approximation.

Case 2: $p \in(1, \infty)$ and $s \in\{0,1\}$. It is well known (see for example [8, Proposition 9.3] for a clear proof) that, for $u \in C_{c}^{\infty}\left(\mathbb{R}^{n}\right)$, we have

$$
\|u(\cdot+y)-u\|_{L^{p}\left(\mathbb{R}^{n}\right)} \leq\|\nabla u\|_{L^{p}\left(\mathbb{R}^{n}\right)}|y| \quad \text { and } \quad\|u(\cdot+y)-u\|_{L^{p}\left(\mathbb{R}^{n}\right)} \leq 2\|u\|_{L^{p}\left(\mathbb{R}^{n}\right)} .
$$

Case 3: $p \in(1, \infty)$ and $s \in(0,1)$. For $y$ fixed, let $T: u \mapsto u(\cdot+y)-u$. Then $T u \in C_{c}^{\infty}\left(\mathbb{R}^{n}\right)$. Now we partially reproduce the proof of [1, Theorem 7.23] (which we state later as Theorem 3.1). We write

$$
\begin{aligned}
K\left(t, T u, L^{p}\left(\mathbb{R}^{n}\right), L^{p}\left(\mathbb{R}^{n}\right)\right) & =\inf \left\{\left\|T u_{0}\right\|_{L^{p}\left(\mathbb{R}^{n}\right)}+t\left\|T u_{1}\right\|_{L^{p}\left(\mathbb{R}^{n}\right)}: u=u_{0}+u_{1}, u_{i} \in L^{p}\left(\mathbb{R}^{n}\right)\right\} \\
& =\inf \left\{\left\|T u-T u_{1}\right\|_{L^{p}\left(\mathbb{R}^{n}\right)}+t\left\|T u_{1}\right\|_{L^{p}\left(\mathbb{R}^{n}\right)}: u_{1} \in L^{p}\left(\mathbb{R}^{n}\right)\right\}
\end{aligned}
$$

Actually, we can consider the infimum over the functions $u_{1} \in C_{c}^{\infty}\left(\mathbb{R}^{n}\right)$, since for a minimising sequence in $L^{p}\left(\mathbb{R}^{n}\right)$ we can construct another minimizing sequence in $C_{c}^{\infty}\left(\mathbb{R}^{n}\right)$ (for example by mollification). This observation together with (3.6) and the definition of $\bar{K}$ given in (3.3) lead to

$$
\begin{aligned}
K\left(t, T u, L^{p}\left(\mathbb{R}^{n}\right), L^{p}\left(\mathbb{R}^{n}\right)\right) & =\inf \left\{\left\|T u-T u_{1}\right\|_{L^{p}\left(\mathbb{R}^{n}\right)}+t\left\|T u_{1}\right\|_{L^{p}\left(\mathbb{R}^{n}\right)}: u_{1} \in C_{c}^{\infty}\left(\mathbb{R}^{n}\right)\right\} \\
& \leq \inf \left\{2\left\|u-u_{1}\right\|_{L^{p}\left(\mathbb{R}^{n}\right)}+t|y|\left\|\nabla u_{1}\right\|_{L^{p}\left(\mathbb{R}^{n}\right)}: u_{1} \in C_{c}^{\infty}\left(\mathbb{R}^{n}\right)\right\} \\
& =2 \inf \left\{\left\|u-u_{1}\right\|_{L^{p}\left(\mathbb{R}^{n}\right)}+\frac{t|y|}{2}\left\|\nabla u_{1}\right\|_{L^{p}\left(\mathbb{R}^{n}\right)}: u_{1} \in C_{c}^{\infty}\left(\mathbb{R}^{n}\right)\right\} \\
& =2 \bar{K}\left(\frac{t|y|}{2}, u\right) .
\end{aligned}
$$


Inserting the previous estimate in the definition of $K$-interpolant norm given in (3.1) and changing variables, we get

$$
\begin{aligned}
\|u(\cdot+y)-u\| \|_{\left(L^{p}\left(\mathbb{R}^{n}\right), L^{p}\left(\mathbb{R}^{n}\right)\right)_{s, p ; K}} & \leq\left(s(1-s) \int_{0}^{+\infty}\left(2 \bar{K}\left(\frac{t|y|}{2}, u\right) \frac{1}{t^{s}}\right)^{p} \frac{\mathrm{d} t}{t}\right)^{\frac{1}{p}} \\
& \leq 2^{1-s}|y|^{s}\left(s(1-s) \int_{0}^{+\infty}\left(\frac{\bar{K}(t, u)}{t^{s}}\right)^{p} \frac{\mathrm{d} t}{t}\right)^{\frac{1}{p}} \\
& =2^{1-s}\|u\|_{s, p}|y|^{s} .
\end{aligned}
$$

This, together with identity (3.5), implies

$$
\begin{aligned}
\|u(\cdot+y)-u\|_{L^{p}\left(\mathbb{R}^{n}\right)} & =p^{\frac{1}{p}}\|u(\cdot+y)-u\|_{\left(L^{p}\left(\mathbb{R}^{n}\right), L^{p}\left(\mathbb{R}^{n}\right)\right)_{s, p ; K}} \\
& \leq p^{\frac{1}{p}} 2^{1-s}\|u\|_{s, p}|y|^{s}
\end{aligned}
$$

From [7, Proposition 4.5] we recover (3.4) with an explicit constant. More precisely,

$$
\|u\|_{s, p} \leq \frac{2 n(n+1)}{\left(n \omega_{n}\right)^{\frac{1}{p}}}[u]_{W^{s, p}\left(\mathbb{R}^{n}\right)}
$$

Hence

$$
\|u(\cdot+y)-u\|_{L^{p}\left(\mathbb{R}^{n}\right)} \leq \frac{2^{2-s} n(n+1) p^{\frac{1}{p}}}{\left(n \omega_{n}\right)^{\frac{1}{p}}}[u]_{W^{s, p}\left(\mathbb{R}^{n}\right)}|y|^{s}
$$

which is precisely (1.2). We can estimate the constant uniformly in $s \in[0,1]$ and $p \in[1, \infty]$ by joining (3.6) and (3.7) to get

$$
\max \left\{2, \frac{2^{2-s} n(n+1) p^{\frac{1}{p}}}{\left(n \omega_{n}\right)^{\frac{1}{p}}}\right\} \leq 4 n(n+1) e^{\frac{1}{e \omega_{n} n}}=: C(n)
$$

Case 4: $p=1$ and $s \in[0,1]$. From the explicit values above, there is a finite limit as $p \rightarrow 1$. By the explicit formula of the Gagliardo seminorm, it is clear that for $u \in C_{c}^{\infty}\left(\mathbb{R}^{n}\right)$ we have $[u]_{W^{s, p}\left(\mathbb{R}^{n}\right)} \rightarrow$ $[u]_{W^{s, 1}\left(\mathbb{R}^{n}\right)}$ as $p \searrow 1$.

Remark 5. Note that in (3.8), we have $n \omega_{n} \rightarrow 0$ as $n \rightarrow \infty$, and thus $C(n)$ diverges as $n \rightarrow \infty$, which does not allow us to get a uniform bound in $n$.

We are now ready to prove our result regarding higher-order Sobolev spaces.

Proof of Theorem 1.2. Let $k=1$. For $u \in \mathcal{C}_{c}^{2}\left(\mathbb{R}^{n}\right)$ we write the Taylor expansion

$$
u(x+y)=u(x)+\int_{0}^{1} \nabla u(x+t y) \cdot y \mathrm{~d} t
$$

Therefore,

$$
u(x+y)-(u(x)+\nabla u(x) \cdot y)=\int_{0}^{1}(\nabla u(x+t y)-\nabla u(x)) \cdot y \mathrm{~d} t .
$$

Integrating over $\mathbb{R}^{n}$, applying Jensen's inequality and Fubini's theorem we have

$$
\begin{aligned}
\int_{\mathbb{R}^{n}}|u(x+y)-(u(x)+\nabla u(x) \cdot y)|^{p} \mathrm{~d} x & \leq \int_{\mathbb{R}^{n}} \int_{0}^{1}|\nabla u(x+t y)-\nabla u(x)|^{p}|y|^{p} \mathrm{~d} t \mathrm{~d} x \\
& \leq|y|^{p} \int_{0}^{1}\|\nabla u(\cdot+t y)-\nabla u\|_{L^{p}\left(\mathbb{R}^{n}\right)}^{p} \mathrm{~d} t \\
& =|y|^{p} \sup _{t \in[0,1]}\|\nabla u(\cdot+t y)-\nabla u\|_{L^{p}\left(\mathbb{R}^{n}\right)}^{p} .
\end{aligned}
$$


Since by definition of the fractional Sobolev spaces, $\nabla u \in W^{s-1, p}\left(\mathbb{R}^{n}\right)^{n}$ we apply Theorem 1.1 to recover

$$
\int_{\mathbb{R}^{n}}|u(x+y)-(u(x)-\nabla u(x) \cdot y)|^{p} \mathrm{~d} x \leq C|y|^{p}|y|^{p(s-1)}\|\nabla u\|_{W^{s-1, p}\left(\mathbb{R}^{n}\right)}^{p} .
$$

The cases when $k>1$ are proved by induction and an analogous argument.

For the reader's convenience we recall an interpolation result that can be found in [1], since we apply part of its proof above.

Theorem 3.1 (Theorem 7.23(a) in [1]). Let $\left\{X_{0}, X_{1}\right\}$ and $\left\{Y_{0}, Y_{1}\right\}$ be two interpolation pairs. Let, for $\theta \in(0,1)$ and $q \in[1, \infty)$

$$
X_{\theta}=\left(X_{0}, X_{1}\right)_{\theta, q ; K}, \quad Y_{\theta}=\left(Y_{0}, Y_{1}\right)_{\theta, q ; K}
$$

with their $K$-interpolation norms. Then, for any $T: X_{0}+X_{1} \rightarrow Y_{0}+Y_{1}$ such that it is linearly continuous $T: X_{i} \rightarrow Y_{i}$ we have that

$$
\|T\|_{\mathcal{L}\left(X_{\theta}, Y_{\theta}\right)} \leq\|T\|_{\mathcal{L}\left(X_{0}, Y_{0}\right)}^{1-\theta}\|T\|_{\mathcal{L}\left(X_{1}, Y_{1}\right)}^{\theta}
$$

\section{Comments and open problems}

1. Some general Rellich-Kondrachov embeddings for different $q$ can also be obtained by interpolation.

2. By extending the argument in Theorem 2.1, it is possible to prove the general Sobolev compact embedding theorem, i.e. possible to prove that $0 \leq t<s$ the space $W^{s, p}\left(\mathbb{R}^{n}\right)$ is compactly embedded in $W^{t, q}(\Omega)$ if $\frac{1}{p}-\frac{s}{n}<\frac{1}{q}-\frac{t}{n}$.

3. In [10] the authors fix a "defect" of the Gagliardo at $s=1$ by replacing the integration by a norm in a Marcinkiewicz space. It is interesting to know if the results in this paper could adapt to those norms.

4. Our results apply to domains $\Omega$, as long as there is an extension operator $E: W^{s, p}(\Omega) \rightarrow W^{s, p}\left(\mathbb{R}^{n}\right)$. A nice discussion of the fractional Sobolev in bounded domains and the existence of this extensions can be found, e.g., in [17].

5. The convergence of the convolution under suitable hypotheses for $\rho_{\varepsilon}$ in Theorem 2.3 for $s>2$ is left open. This kind of result have been studied for $p=2$ by Fourier transform.

6. Discretizations of nonlocal operators like the fractional Laplacian $(-\Delta)^{s}$ based on Lagrange interpolants (see [15, Section 4.4]) can be shown to have a consistency error in $L^{p}\left(\mathbb{R}^{n}\right)$ of order $h^{\sigma-2 s}$ for $W^{\sigma, p}\left(\mathbb{R}^{n}\right)$. This topic will be thoroughly studied in the forthcoming paper [16].

7. When $s=\{0,1\}$, the constant in Theorem 1.1 does not depend on the dimension either. We wonder if the dependence on $n$ can be dropped also when $s \in(0,1)$ (see Remark 5). More generally, the calculation of the optimal constant for $s \in(0,1)$ and variable $p$ is an important question.

\section{Acknowledgements}

F. del Teso was partially supported by PGC2018-094522-B-I00 from the MICINN, of the Spanish Government. The work of D. Gómez-Castro and J. L. Vázquez were funded by grant PGC2018-098440-B-I00 from the MICINN, of the Spanish Government. J. L. Vázquez is an Honorary Professor at Univ. Complutense de Madrid. The authors are thankful to L. Brasco for information and useful comments. 


\section{References}

[1] R. Adams and J. Fournier. Sobolev spaces. Vol. 140. Academic press, 2003.

[2] H. Amann. Compact embedding of vector-valued Sobolev and Besov spaces. Glasnik Matematicki 35.55 (2000), pp. $161-177$.

[3] L. Ambrosio, G. de Philippis, and L. Martinazzi. Gamma-convergence of nonlocal perimeter functionals. Manuscripta Mathematica 134.3 (2011), pp. 377-403. DOI: 10.1007/s00229-010-0399-4. arXiv: 1007.3770 .

[4] C. Bennet and R. Sharpley. Interpolation of Operators. New York: Academic Press, 1988.

[5] J. Bourgain, H. Brezis, and P. Mironescu. "Another look at Sobolev spaces". Optimal Control and Partial Differential Equations. Amsterdam: IOS Press, 2001, pp. 439-455.

[6] L. Brasco, E. Lindgren, and E. Parini. The fractional Cheeger problem. Interfaces and Free Boundaries 16.3 (2014), pp. 419-458. DOI: 10.4171/IFB/325.

[7] L. Brasco and A. Salort. A note on homogeneous Sobolev spaces of fractional order. Annali di Matematica Pura ed Applicata 198.4 (2019), pp. 1295-1330. DOI: 10.1007/s10231-018-0817-x. arXiv: 1806.08945.

[8] H. Brezis. Functional Analysis, Sobolev Spaces and Partial Differential Equations. New York: Springer, 2010. DOI: 10.1007/978-0-387-70914-7.

[9] H. Brezis and P. Mironescu. Gagliardo-Nirenberg inequalities and non-inequalities: The full story. Annales de l'Institut Henri Poincare (C) Analyse Non Lineaire 35.5 (2018), pp. 1355-1376. DOI: $10.1016 / j$.anihpc.2017.11.007.

[10] H. Brezis, J. V. Schaftingen, and P.-l. Yung. "A surprising formula for Sobolev noms and related topics". To appear.

[11] O. Ciaurri, L. Roncal, P. R. Stinga, J. L. Torrea, and J. L. Varona. Nonlocal discrete diffusion equations and the fractional discrete Laplacian, regularity and applications. Adv. Math. 330 (2018), pp. 688-738. DOI: 10.1016/j.aim.2018.03.023.

[12] N. A. Dao, J. I. DíAz, and Q. H. NGuYen. Generalized Gagliardo-Nirenberg inequalities using Lorentz spaces, BMO, Hölder spaces and fractional Sobolev spaces. Nonlinear Analysis, Theory, Methods and Applications 173 (2018), pp. 146-153. DOI: 10.1016/j.na.2018.04.001.

[13] N. A. DAo, J. I. DíAz, and Q.-H. NGUYEN. Fractional Sobolev inequalities revisited: the maximal function approach. Rendiconti Lincei - Matematica e Applicazioni 31.1 (2020), pp. 225-236. DOI: $10.4171 /$ RLM/887.

[14] J. DÁvila. On an open question about functions of bounded variation. Calculus of Variations and Partial Differential Equations 15.4 (2002), pp. 519-527. DOI: 10.1007/s005260100135.

[15] F. Del Teso, J. Endal, and E. R. Jakobsen. Robust numerical methods for nonlocal (and local) equations of porous medium type. Part II: Schemes and experiments. SIAM J. Numer. Anal. 56.6 (2018), pp. 3611-3647. DOI: 10.1137/18M1180748.

[16] F. Del Teso, D. Gómez-Castro, and J. L. Vázquez. Robust finite difference schemes for the fractional dirichlet problem. In preparation (2020).

[17] E. Di Nezza, G. Palatucci, and E. Valdinoci. Hitchhiker's guide to the fractional Sobolev spaces. Bulletin des Sciences Mathematiques 136.5 (2012), pp. 521-573. DOI: 10.1016/j . bulsci.2011.12.004. arXiv: 1104.4345.

[18] I. Drelichman and R. G. DuRÁn. Improved Poincaré inequalities in fractional Sobolev spaces. Annales Academiae Scientiarum Fennicae Mathematica 43 (2018), pp. 885-903. DOI: 10.5186/AASFM. 2018.4352. arXiv: 1705.04227.

[19] G. Leoni. A First Course in Sobolev Spaces. Vol. 105. Graduate Studies in Mathematics. Providence, Rhode Island: American Mathematical Society, 2009, p. 626. DoI: 10.1090/gsm/105.

[20] V. MaZ'ya and T. Shaposhnikova. On the Bourgain, Brezis, and Mironescu theorem concerning limiting embeddings of fractional Sobolev spaces. Journal of Functional Analysis 195.2 (2002), pp. 230-238. DOI: $10.1006 / \mathrm{jfan} .2002 .3955$. 
[21] V. MaZ'Ya. Sobolev Spaces. Vol. 342. Grundlehren der mathematischen Wissenschaften. Berlin, Heidelberg: Springer Berlin Heidelberg, 2011. DOI: 10.1007/978-3-642-15564-2.

[22] M. Milman. Notes on limits of Sobolev spaces and the continuity of interpolation scales. Transactions of the American Mathematical Society 357.9 (2005), pp. 3425-3442. DOI: 10.1090/S0002-9947-05-03937-1.

[23] J. Simon. Compact Sets in the space $L^{p}(0, T ; B)$. Annali di Matematica pura ed applicata 146.65-96 (1986).

[24] L. TARTAR. An Introduction to Sobolev Spaces and Interpolation Spaces. Lecture Notes of the Unione Matematica Italiana. Berlin, Heidelberg: Springer Berlin Heidelberg, 2007. DOI: 10.1007/978-3-540-71483-5. 